\title{
Interstitial Pneumonia in a 94-Year-0ld Woman with MPO-ANCA Positive Vasculitis
}

\author{
Daisuke Usuda1,2, Ryusho Sangen1, Yoshifumi Iwata1, Tsugiyasu Kanda1 ${ }^{*}$ \\ ${ }^{1}$ Department of Community Medicine, Kanazawa Medical University Himi Municipal Hospital, Himi, Japan \\ ${ }^{2}$ Department of Infectious Diseases, Kanazawa Medical University, Uchinada, Japan \\ Email: "kandat@kanazawa-med.ac.jp
}

Received 19 December 2015; accepted 21 February 2016; published 24 February 2016

Copyright (C) 2016 by authors and Scientific Research Publishing Inc.

This work is licensed under the Creative Commons Attribution International License (CC BY).

http://creativecommons.org/licenses/by/4.0/

c) (i) Open Access

\begin{abstract}
The interstitial pneumonia (IP) associated with myeloperoxidase anti-neutrophil cytoplasmic autoantibody (MPO-ANCA) showed characteristic histology dominated by usual IP pattern. We showed a 94-year-old woman, whose prognosis was poor by severity of IP by MPO-ANCA associated vasculitis. The median age at diagnosis of IP by MPO-ANCA associated vasculitis was over 60 years, with a male predominance. Most patients died related to respiratory insufficiency. Our case would be the oldest patient in IP by MPO-ANCA associated vasculitis in the literature.
\end{abstract}

Keywords

Interstitial Pneumonia, MPO-ANCA, Woman, Oldest

\section{Introduction}

The interstitial pneumonia (IP) associated with myeloperoxidase anti-neutrophil cytoplasmic autoantibody (MPO-ANCA) showed characteristic histology dominated by usual IP pattern. IP associated with MPO-ANCA may be a histologically distinctive disease from idiopathic pulmonary fibrosis. Mortality was relatively high and life threatening acute exacerbation may occur. The age of patients in IP associated with MPO-ANCA may be related poor prognostic course, since they suffer the other significant sickness and their symptoms may be masked by their holding diseases [1]-[3].

The natural history and pathogenesis of IP are controversial, and ongoing research continues to investigate multiple hypotheses. IP generally occurs after the age of 60 years and is more prevalent in men. The initial clinical presentation is not specific, consisting of progressive dyspnoea on exertion combined with dry cough. Its prevalence and incidence still remain unclear, with a large variability across reports due to a number of reasons.

*Corresponding author.

How to cite this paper: Usuda, D., Sangen, R., Iwata, Y. and Kanda, T. (2016) Interstitial Pneumonia in a 94-Year-Old Woman with MPO-ANCA Positive Vasculitis. Case Reports in Clinical Medicine, 5, 61-65.

http://dx.doi.org/10.4236/crcm.2016.52011 
The majority of the studies have showed higher prevalence and incidence rates among men and with increasing age, especially after 75 years. Older age and male sex seem to be correlated with a poorer prognosis in IF patients, whereas there are contrasting data about smoking status [4].

We show a 94-year-old woman with IP associated MPO-ANCA and discuss the relation between elderly patients and the disease characters.

\section{Case Presentation}

A 94-year-old woman presented with a 4-day history of exertional dyspnea and productive cough. She quitted smoking 20 years ago. She was treated with chronic renal failure, dementia and lipid disorder in an open clinic. Her examination revealed facial and pitting edema. Respiratory examination revealed fine bilateral end-inspiratory crepitations. Initial investigation revealed raised blood inflammatory markers (C reactive protein; 4.90 $\mathrm{mg} / \mathrm{dL}$ ) and renal dysfunctin (creatinine; $2.59 \mathrm{mg} / \mathrm{dL}$ ) and MPO-ANCA was positive of $15.8 \mathrm{IU} / \mathrm{ml}$ with the normal range being $0-3.5 \mathrm{IU} / \mathrm{ml}$. The proteinase 3(PR3)-ANCA was negative (5 IU/ml). The titer of antinuclear antibody was slightly positive, such as 40 times. The level of serum KL-6, mucin-associated antigen, was positive of 62,600 U/ml. Other serum biomarkers such as SP-A, SP-D were not measured. Urine dipstick and microscopy revealed microscopic haematuria, glass casts and proteinuria. The chest $\mathrm{X}$-ray showed congestion and cardiac enlargement with interstitial fibrosis (Figure 1). The chest radiograph showed basal pulmonary fibrosis with usual interstitial pneumonia pattern on high-resolution computed tomography (CT) imaging (Figure 2). The CT scans were reviewed in a blinded fashion by a chest radiologist experienced in the interpretation of diffuse lung disease and were classified using the radiologic patterns described in the ATS/ERS International Consensus Classification 2002 of the Idiopathic Interstitial Pneumonias (IIPs) [4]. These clinical and laboratory findings pointed towards an MPO-ANCA associated vasculitis with no features to suggest an infectious cause or alternative connective tissue disorder. This patient was started on 3 days pulse of methly prednisolone at $500 \mathrm{mg} \mathrm{mg} /$ day intravenously. She did not improve from respiratory symptoms. Finally, she became multiple organ failure and died at 26 days after admission.

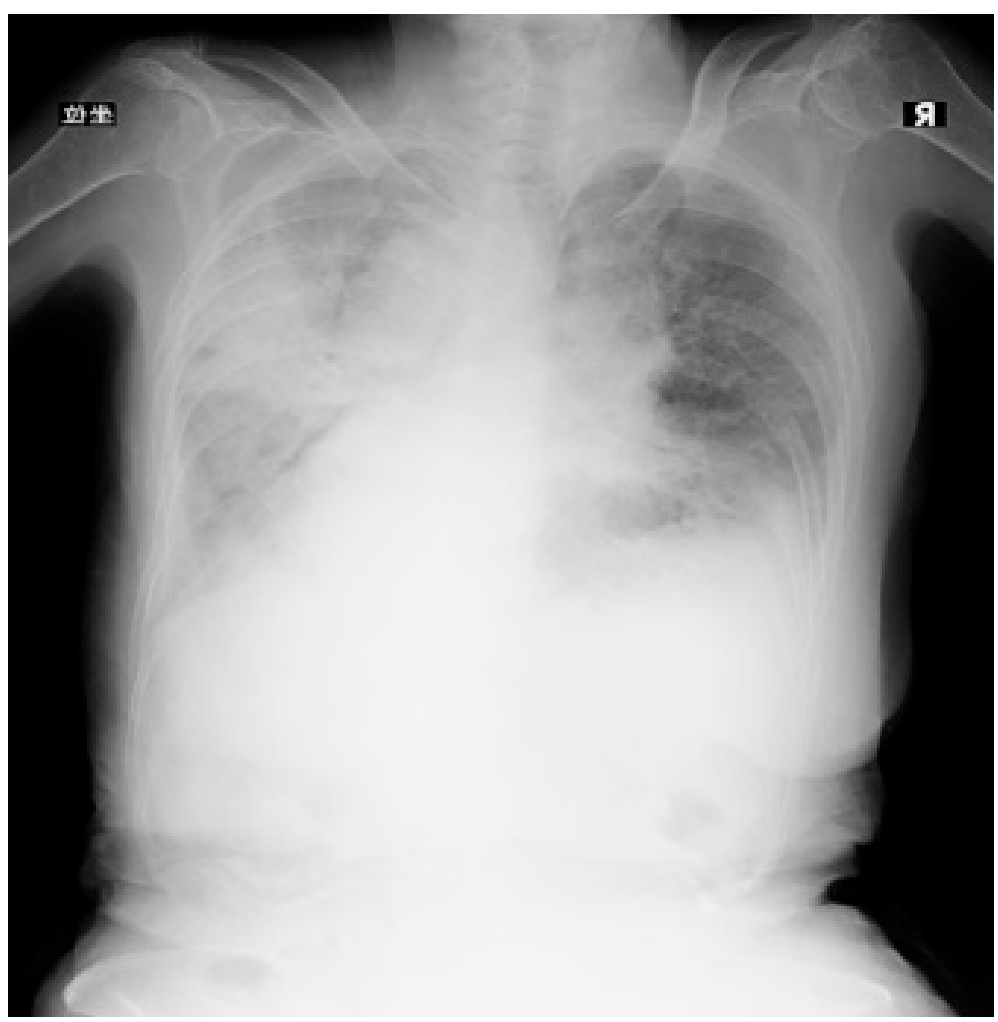

Figure 1. Chest X-ray in admission revealed congestion and cardiac enlargement with interstitial fibrosis. 


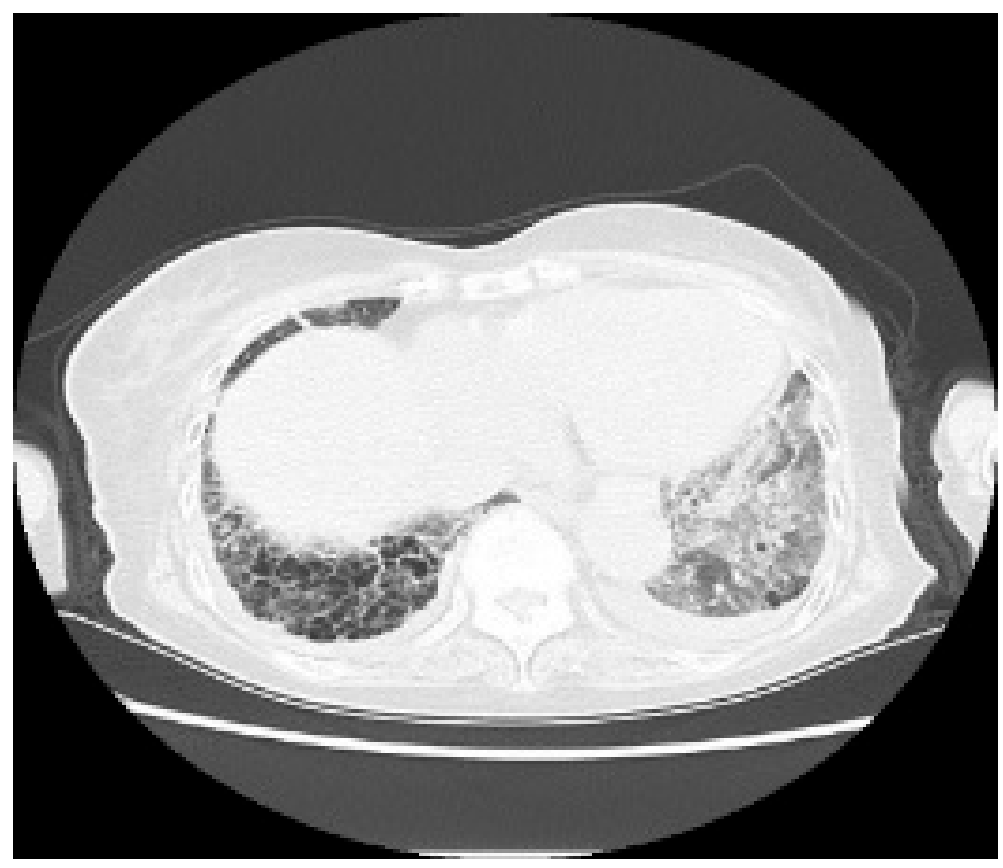

Figure 2. High-resolution CT scan showing classical features of usual interstitial pneumonitis with subpleural basal honeycomb change with reticular abnormality.

\section{Discussion}

We showed a 94-year-old woman, whose prognosis was poor by severity of IP by MPO-ANCA associated vasculitis. Mortality was relatively high and life threatening acute exacerbation may occur. The previous reports show that median age at diagnosis of IP by MPO-ANCA associated vasculitis was over 60 (range; 4 - 85) years, with a male predominance [1]-[3]. Most patients died related to respiratory insufficiency. Our case would be the oldest patient in IP by MPO-ANCA associated vasculitis in the literature.

MPO-ANCA is a well-known marker for small vessel vasculitis. Recent reports have demonstrated that IP may rarely be associated with serum MPO-ANCA. Mortality was high and life threatening acute exacerbation may occur. According to the guideline for IP [5], basically all IP cases are recommended to examine serum ANA, MPO-ANCA, PR3-ANCA and other autoimmune antibody as a routine screening test to exclude the collagen vascular disease from IIPs. In the elderly, other symptoms such as hearing loss, recognition impairment, and cerebral infarction often disturb the definite diagnosis which will be taken several months [2]. Therefore, improving awareness and vigilance regarding this disease is important for early diagnosis and treatment.

Although the relationship between the clinical damages and patients' age and course of disease revealed that age and disease course do not have a significant effect on the kidney, and heart involvement, the age of patients with pulmonary damage was significantly higher than that of patients without lung damage, and the course of disease was shorter, which suggested that elderly patients experienced early lung damage, or more serious lung damage such as pulmonary hemorrhage leading to early death [5]. Older patients were more likely to show lung involvement in the early disease stage in Japan [6]. This tendency is compatible with European patients [2].

Elderly patients in IP with MPO-ANCA are predominantly MPO-ANCA positive compared to PR3 ANCA. IP with MPO-ANCA in elderly frequently involves the kidney [7]. The elderly patients present more often with severe renal disease and have more infections as a consequence of immunosuppressive therapy and higher mortality [8]. The combination of older age and renal insufficiency portends a poor prognosis due to poor response to therapy and increased therapy related adverse events. Observational studies of ANCA-associated vasculitis in the elderly, have demonstrated a poorer prognosis for older patients, with significantly higher rates of death and treatment-related complications [9]. Following these reports, this case showed multiple organ failure after prednisolone administration. With the anticipated doubling of Japanese aged 65 or older in the next 25 years [10] and the predilection of IP with MPO-ANCA associated vasculitis for elderly, our ability to identify safer alternatives 
to standard of care therapy in this vulnerable population becomes paramount.

The authors conclude that the age-related changes of various immunological parameters are different between men and women, likely due to a lower biological age of women [11]. These findings, indicating a slower rate of decline in these immunological parameters in women than in men, are consistent with the fact that women live longer than men, i.e. in Japan 85.5 years in women and 79.0 in men [10]. A sexual dimorphism in the immune response means that females are more resistant to infections but they have higher incidence of autoimmune diseases compared to male [12]. This aged woman revealed poor prognosis from IP with MPO-ANCA associated vasculitis, probably due to immunological impairments.

\section{Conclusion}

The authors conclude that aged woman revealed poor prognosis from IP with MPO-ANCA associated vasculitis, probably due to immunological impairments. Older patients are more likely to show lung involvement in the early disease stage of IP with MPO-ANCA associated vasculitis. Physicians should be aware of secondary IP with autoimmune diseases in very old patients instead of infection and malignancy.

\section{Disclaimer}

The views expressed herein are those of the authors and do not reflect the official policy orposition of the Kanazawa Medical University Himi Municipal Hospital, Department of Community Medicine.

\section{Conflict of Interests}

The authors declare that there is no conflict of interests regarding the publication of this paper.

\section{Ethical Approval}

This study was conducted with the approval of the ethics committee of Kanazawa Medical University Himi Municipal Hospital.

\section{References}

[1] Comarmond, C., Crestani, B., Tazi, A., et al. (2014) Pulmonary Fibrosis in Antineutrophil Cytoplasmic Antibodies (ANCA)-Associated Vasculitis: A Series of 49 Patients and Review of the Literature. Medicine (Baltimore), 93, 340-349. http://dx.doi.org/10.1097/MD.0000000000000217

[2] Shuai, Z.W., Lv, Y.F., Zhang, M.M. and Hu, Z.Y. (2015) Clinical Analysis of Patients with Myeloperoxidase Antineutrophil Cytoplasmic Antibody-Associated Vasculitis. Genetics and Molecular Research, 14, 5296-5303. http://dx.doi.org/10.4238/2015.May.18.22

[3] Andreiana, I., Stancu, S., Avram, A., Taran, L. and Mircescu, G. (2015) ANCA Positive Crescentic Glomerulonephritis Outcome in a Central East European Cohort: A Retrospective Study. BMC Nephrology, 16, 90. http://dx.doi.org/10.1186/s12882-015-0091-8

[4] Sgalla, G., Biffi, A. and Richeldi, L. (2015) Idiopathic Pulmonary Fibrosis: Diagnosis, Epidemiology and Natural History. Respirology. http://dx.doi.org/10.1111/resp.12683

[5] Demedts, M. and Costabel, U. (2002) ATS/ERS International Multidisciplinary Consensus Classification of Idiopathic Interstitial Pneumonias. General Principles and Recommendations. European Respiratory Journal, 19, 794-796. http://dx.doi.org/10.1183/09031936.02.00492002

[6] Tanaka, T., Otani, K., Egashira, R., et al. (2012) Interstitial Pneumonia Associated with MPO-ANCA: Clinicopathological Features of Nine Patients. Respiratory Medicine, 106, 1765-1770. http://dx.doi.org/10.1016/j.rmed.2012.08.024

[7] Bomback, A.S., Appel, G.B., Radhakrishnan, J., et al. (2011) ANCA-Associated Glomerulonephritis in the Very Elderly. Kidney International, 79, 757-764. http://dx.doi.org/10.1038/ki.2010.489

[8] Harper, L. and Savage, C.O. (2005) ANCA-Associated Renal Vasculitis at the End of the Twentieth Century-A Disease of Older Patients. Rheumatology (Oxford), 44, 495-501. http://dx.doi.org/10.1093/rheumatology/keh522

[9] Manno, R.L., Seo, P. and Geetha, D. (2015) Older Patients with ANCA-Associated Vasculitis and Dialysis Dependent Renal Failure: A Retrospective Study. BMC Nephrology, 16, 88. http://dx.doi.org/10.1186/s12882-015-0082-9

[10] Sumi, K., Kawakubo, Y., Yamashita, Y., Goto, M. and Kuraoka, A. (2014) Generational Differences in Tooth Size in the Japanese Population: Analysis of Cohorts with a Generation Gap of Four to Five Decades. Fukuoka Igaku Zasshi, 
105, 225-233.

[11] Caruso, C., Candore, G., Romano, G.C., et al. (2001) Immunogenetics of Longevity. Is Major Histocompatibility Complex Polymorphism Relevant to the Control of Human Longevity? A Review of Literature Data. Mechanisms of Ageing and Development, 122, 445-462. http://dx.doi.org/10.1016/S0047-6374(00)00255-4

[12] Hirokawa, K., Utsuyama, M., Hayashi, Y., et al. (2013) Slower Immune System Aging in Women versus Men in the Japanese Population. Immunity \& Ageing, 10, 19. http://dx.doi.org/10.1186/1742-4933-10-19 